\title{
Analysis of Internal Energy Transfer Within a Modular Particle-Continuum Method
}

\author{
Timothy R. Deschenes*, Timothy D. Holman`, and Iain D. Boyd ${ }^{\ddagger}$ \\ Department of Aerospace Engineering, University of Michigan, Ann Arbor, MI, 48109 \\ and \\ Thomas E. Schwartzentruber ${ }^{\S}$ \\ Department of Aerospace Engineering and Mechanics, University of Minnesota, Minneapolis, MN, 55455
}

\begin{abstract}
A modular particle-continuum (MPC) method is extended to include internal energy nonequilibrium to simulate hypersonic steady-state flows that exhibit small regions of collisional nonequilibrium in a mainly continuum flow field. This method loosely couples an existing direct simulation Monte Carlo (DSMC) code to a Navier-Stokes solver (CFD) while allowing both time-step and cell size to be completely decoupled between each method. By limiting the size of the DSMC region to areas in collisional nonequilibrium, the MPC method is able to reproduce full DSMC results to within $5 \%$ while decreasing the computational time required by factors of 3 to 5 . The goal of the present study is to incorporate rotational excitation in the hybrid method so that the size of the DSMC domain can be reduced. In addition, two vibrational relaxation methods are tested in the DSMC method. Results from full DSMC, full CFD, and the hybrid method are compared for both relaxation methods. It is found that having compatible relaxation models slightly improves flow field results. Further work on an adequate breakdown parameter for the prediction of collisional nonequilibrium may improve the accuracy and efficiency of the hybrid code.
\end{abstract}

\section{Nomenclature}

$C_{h} \quad$ Heat transfer coefficient

$C_{p} \quad$ Surface pressure coefficient

$d \quad$ Diameter $[m]$

$E \quad$ Energy per unit volume $\left[\frac{J}{\mathrm{~m}^{3}}\right]$

$f \quad$ Probability density function

$g \quad$ Relative speed $\left[\frac{m}{s}\right]$

$k \quad$ Boltzmann constant $\left[1.38 \times 10^{-23} \frac{J}{K}\right]$

$m \quad$ Molecular mass $[\mathrm{kg}]$

$n \quad$ Number density $\left[\frac{1}{m^{3}}\right]$

$p \quad$ Pressure $[\mathrm{Pa}]$

$P \quad$ Average probability

$Q \quad$ Flow quantity

$\vec{q} \quad$ Heat flux $\left[\frac{W}{m^{2}}\right]$

$S \quad$ Internal relaxation source term

$T \quad$ Temperature $[K]$

u Velocity $\left[\frac{\mathrm{m}}{\mathrm{s}}\right]$

$w \quad$ Source term

Z Collision number

*Graduate Student, Student Member AIAA. Email: thytimo@umich.edu.

${ }^{\dagger}$ Graduate Student, Student Member AIAA. Email: tholman@umich.edu.

${ }^{\ddagger}$ Professor, Associate Fellow AIAA. Email: iainboyd@umich.edu.

$\S$ Assistant Professor, AIAA Member. Email: schwartz@aem.umn.edu. 


$\begin{array}{ll}\eta & \text { Number of internal degrees of freedom } \\ \lambda & \text { Mean free path }[\mathrm{m}] \\ \mu & \text { Coefficient of viscosity }\left[\frac{\mathrm{kg}}{\mathrm{ms}}\right], \text { reduced mass }[\mathrm{kg}] \\ \nu & \text { Mean collision frequency } \\ \Phi & \text { Subrelaxation weight } \\ \phi & \text { Variable probability } \\ \rho & \text { Mass density }\left[\frac{\mathrm{kg}}{\mathrm{m}^{3}}\right] \\ \theta & \text { Characteristic temperature }[\mathrm{K}] \\ \sigma & \text { Reference cross section }\left[\mathrm{m}^{2}\right] \\ \tau & \text { Relaxation time }[\mathrm{s}] \\ \omega & \text { VHS viscosity temperature exponent } \\ & \\ \text { Subscripts } \\ C F D & \text { Computational Fluid Dynamics } \\ D S M C & \text { direct simulation Monte Carlo } \\ M W & \text { Millikan and White } \\ P & \text { Park } \\ r e f & \text { Reference } \\ \text { rot } & \text { Rotational } \\ s & \text { Species } \\ \text { tra } & \text { Translational } \\ v i b & \text { Vibrational } \\ \infty & \text { Free stream }\end{array}$

\section{Introduction}

As a hypersonic vehicle enters a planetary atmosphere, the variation of characteristic flow length and time scales about the vehicle leads to a multi-scale gas flow. One parameter used to compare the variation in length scales is the Knudsen number. At sufficiently low Knudsen numbers, many gas particle collisions occur around the body and the flow can be considered in collisional equilibrium. When the flow is considered near collisional equilibrium, continuum flow formulations such as the Navier-Stokes equations provide a physically accurate description of the flow field. Computational Fluid Dynamics (CFD) provides accurate and efficient numerical solutions to the Navier-Stokes equations. However, at high Knudsen numbers, the flow can be considered rarefied, or in collisional nonequilibrium. In this regime, the approximations used to derive the Navier-Stokes equations break down and the flow can only be accurately described using a kinetic description. The direct simulation Monte Carlo (DSMC) method $^{1}$ first proposed by Bird provides an approach that can accurately model dilute gas flows described by the Boltzmann equation. Though physically accurate over all flow conditions, DSMC becomes prohibitively expensive at higher densities due to time and length scale restrictions set by separating the move and collision processes in the method. At these higher densities, the approximations used to form the Navier-Stokes equations are valid, and the flow can be computed using CFD. For many hypersonic flows of interest, some regions of the flow field can be sufficiently rarefied so that CFD can not be used over the entire flow field while simultaneously having regions that can be considered well within the continuum regime making full DSMC simulations prohibitively expensive. Instead, a hybrid method can be employed that takes advantage of the computational efficiency of CFD in regions that are near collisional equilibrium, while maintaining the physical accuracy of DSMC in regions that are rarefied.

Previous work has been performed using a "zonally decoupled" DSMC-CFD simulation. ${ }^{2-4}$ For these hybrid methods, a CFD solution is calculated to a predetermined interface, then this information is used as the boundary condition for the DSMC simulation over the remainder of the domain. This method is only valid when the rarefied region is completely down stream of the continuum region with no recirculation occurring across the interface. This is because the CFD solution is completely decoupled from the DSMC solution. Often, rarefied regions are highly localized and two way coupling is required to obtain an efficient, physically accurate simulation. Various methods have been proposed that adaptively re-position the interface between DSMC and CFD throughout the simulation using different coupling methods between the two domains..$^{5-8}$ 
Reference 9 presents a discussion of the major considerations involved in coupling a kinetic scheme with a continuum method as well as a summary of published work on past methods. ${ }^{9}$

The present article extends the modular particle-continuum (MPC) method that was first developed for 1-D shock waves ${ }^{10}$ and later extended to 2 -D and axi-symmetric flows. ${ }^{11,12}$ In previous work, the MPC method was able to reproduce full DSMC results. By limiting the DSMC method to only regions that are rarefied, the MPC method can achieve speed-up factors exceeding 3 for transitional flows ${ }^{13}$ and 13 for near continuum flows. ${ }^{12}$ In addition, previous work was performed to increase the physical accuracy of the MPC method at higher enthalpies by including vibrational nonequilibrium. ${ }^{14}$ That work found that the agreement in vibrational temperature between the MPC method and full DSMC was highly dependent on the agreement between relaxation methods used in the CFD and DSMC modules.

This article outlines the extension of the method by including rotational relaxation within both the CFD and DSMC modules of the MPC method. By including rotational nonequilibrium within the CFD module, the regions that can be solved with CFD can be expanded due to the increase in physical accuracy of the CFD module. ${ }^{14,15}$ This, in turn, decreases the size of the DSMC region which leads to a direct decrease in the computational expense of the MPC method. In addition, two different vibrational relaxation methods are used within the DSMC method. Assessment of agreement between the MPC method and full DSMC using each vibrational method is performed. Heat transfer and computational speed-up are presented for simulations employing different physical models in the flow modules. Finally, conclusions are formulated based on the current results and areas of future work are discussed.

\section{Flow Modules}

The modular particle-continuum code (MPC) used in this study was developed to be capable of simulating one-dimensional shock waves ${ }^{10}$ and both axi-symmetric and two dimensional hypersonic flows. ${ }^{11,16}$ It uses the LeMANS ${ }^{17,18}$ code for the continuum regions, while using the DSMC code, MONACO, ${ }^{19}$ to simulate the rarefied regions.

LeMANS is capable of simulating 2-D, axi-symmetric, or 3-D steady-state, continuum, hypersonic flows using a finite volume formulation of the Navier-Stokes equations with the added ability to account for vibrational and rotational nonequilibrium. The inviscid fluxes are solved using a modified form of the Steger-Warming Flux Vector Splitting method which is less dissipative and can be used to calculate boundary layers. The method switches back to the original form of Steger-Warming near shock waves. The viscous terms are calculated using properties at cell nodes and cell centers. The no-slip condition is applied to wall boundaries while the inflow and outflow are treated as supersonic flow. Time integration is performed using a point-implicit method. It can also simulate multi-species and weakly ionized flows with finite rate chemistry, but those models are not used in this study. The Variable Hard Sphere (VHS) model is used to calculate the viscosity-temperature relation that can be formulated using Eqs. 1 and 2 where $\omega, \mu_{\text {ref }}$, and $T_{r e f}$ are constants and $d_{r e f}$ is the species reference diameter. ${ }^{1}$ All results presented within this paper simulate molecular nitrogen with a reference diameter of $d_{r e f}=4.17 \times 10^{-10} \mathrm{~m}$ at $T_{\text {ref }}=273 \mathrm{~K}$ with a power law exponent of $\omega=0.75$.

$$
\begin{gathered}
\mu=\mu_{\text {ref }}\left(\frac{T_{\text {tra }}}{T_{r e f}}\right)^{\omega} \\
\mu_{\text {ref }}=\frac{15 \sqrt{\pi m k T_{r e f}}}{2 \pi d_{r e f}^{2}(5-2 \omega)(7-2 \omega)}
\end{gathered}
$$

MONACO is a general, cell-based implementation of the DSMC method capable of simulating rotational and vibrational nonequilibrium and multi-species flow with finite rate chemistry. The VHS collision model that replicates the macroscopic viscosity-temperature dependence is used. The proceeding subsections describe and compare the internal relaxation processes modeled within both the CFD and DSMC modules.

\section{II.A. Rotational Relaxation}

Both the CFD and DSMC modules have the capability of simulating rotational nonequilibrium. ${ }^{20}$ For the CFD module, a separate rotational energy equation is added to the total system of equations solved. Equation 3 shows the time rate of change of rotational energy per unit volume where $\vec{q}_{\text {rot }}$ is the rotational 
heat flux given by Fourier's law, $\vec{u}_{d s}$ is the species diffusion velocity given by Fick's law and $\dot{w}_{r o t}$ is the rotational energy source term.

$$
\begin{aligned}
\frac{\partial E_{r o t}}{\partial t}+\nabla \cdot\left(E_{r o t} \vec{u}\right) & =-\nabla \cdot\left(\vec{q}_{r o t}\right)-\nabla \cdot \sum_{s}\left(\rho_{s} e_{r o t, s} \vec{u}_{d s}\right)+\dot{w}_{r o t} \\
\dot{w}_{r o t} & =\sum_{s}\left(S_{r o t, s}^{t r a-r o t}+\dot{w}_{s} e_{r o t, s}\right)
\end{aligned}
$$

Equation 4 shows that the rotational source term can be split into two parts: a rotation-translation relaxation source and a source due to the gain and loss of rotational energy through chemical reactions. For this study of a single species gas, the chemistry source is always zero. The rotation-translation relaxation source can be written as Eq. 5 where $e_{r o t, s}^{*}$ is the specific rotational energy based on the translational temperature, $e_{r o t, s}$ is the current specific rotational energy, $Z_{r o t, s}$ is the rotational collision number, and $\tau_{\text {coll }, s}$ is the mean collision time. The rotational collision number shown in Eq. 6, is derived from Parker. ${ }^{21}$ The mean collision frequency, which is the inverse of the mean collision time, for the VHS model given by Bird $^{1}$ is shown in Eq. 7.

$$
\begin{gathered}
S_{\text {rot }, s}^{\text {tra-rot }}=\rho_{s} \frac{e_{\text {rot }, s}^{*}-e_{\text {rot }, s}}{\tau_{\text {rot }, s}}=\rho_{s} \frac{e_{\text {rot }, s}^{*}-e_{\text {rot }, s}}{Z_{\text {rot }, s} \tau_{\text {coll }}} \\
Z_{r o t, s}=\frac{Z_{r o t, s}^{\infty}}{1+\frac{\pi^{\frac{3}{2}}}{2}\left(\frac{T_{s}^{*}}{T_{\text {tra }}}\right)^{\frac{1}{2}}+\left(\frac{\pi^{2}}{4}+\pi\right)\left(\frac{T_{s}^{*}}{T_{\text {tra }}}\right)} \\
\nu_{s}=\frac{1}{\tau_{\text {coll }, s}}=\sum_{i}\left(n_{i} d_{r e f, s, i}^{2}\left(\frac{8 \pi k T_{r e f, i}}{\mu_{s, i}^{*}}\right)^{\frac{1}{2}}\left(\frac{T_{\text {tra }}}{T_{\text {ref }, i}}\right)^{1-\omega_{i}}\right)
\end{gathered}
$$

MONACO uses the variable rotational energy exchange probability model of Boyd ${ }^{22}$ that is also based on Parker. ${ }^{21}$ This formulation uses a probability of a rotationally inelastic collision, $\phi_{\text {rot }, s}\left(E_{c}\right)$, that reproduces the macroscopic relaxation time by satisfying Eq. 8 where $E_{c}$ is the total collisional energy between the two particles and $f\left(E_{c}\right)$ is the collisional energy probability distribution function. Lumpkin et al. found that the definitions of continuum and particle relaxation times differ by a factor given by Eq. 9 where $\eta_{\text {int,tra }}$ is the number of internal degrees of freedom based on the translational temperature and $\omega$ is the VHS temperature exponent. Figure 1(a) compares the predicted temperatures of an adiabatic rotational relaxation process from both CFD and DSMC. Despite differences in modeling the relaxation process, results from the two methods are nearly identical.

$$
\begin{aligned}
\bar{P}_{r o t, s}=\frac{1}{Z_{\text {rot }, s}}= & \frac{1}{\nu_{s} \tau_{\text {rot }, s}}=\int_{0}^{\infty} \phi_{\text {rot }, s}\left(E_{c}\right) f\left(E_{c}\right) d\left(E_{c}\right) \\
\tau_{\text {int }}^{D S M C} & =\frac{\tau_{\text {int }}^{C F D}}{1+\frac{\eta_{\text {int, tra }}}{4-2 \omega}}
\end{aligned}
$$

\section{II.B. Vibrational Relaxation}

In addition to rotational nonequilibrium, both MONACO and LeMANS have the capability of simulating vibrational nonequilibrium. For the CFD module, a separate vibrational energy is added to the total system of equations solved. Equation 10 shows the time rate of change of vibrational energy per unit volume where $\vec{q}_{v i b}$ is the vibrational heat flux given by Fourier's law, $\vec{u}_{d s}$ is the species diffusion velocity given by Fick's law, and $\dot{w}_{v i b}$ is the vibrational source term.

$$
\begin{aligned}
\frac{\partial E_{v i b}}{\partial t}+\nabla \cdot\left(E_{v i b} \vec{u}\right) & =-\nabla \cdot\left(\vec{q}_{v i b}\right)-\nabla \cdot \sum_{s}\left(\rho_{s} e_{v i b, s} \vec{u}_{d s}\right)+\dot{w}_{v i b} \\
\dot{w}_{v i b} & =\sum_{s}\left(S_{v i b, s}^{t r a-v i b}+\dot{w}_{s} e_{v i b, s}\right)
\end{aligned}
$$


The vibrational source term can be split into two parts: a vibrational-translational relaxation source and a source due to the gain and loss of vibrational energy through chemical reactions as shown in Eq. 11. Again, for this work the chemistry source term is always zero. The vibration-translation relaxation source term can be written as Eq. 12 where $e_{v i b, s}^{*}$ is the specific vibrational energy evaluated at the translational temperature and $\tau_{v i b}$ is the vibration-translation relaxation time. This relaxation time can be calculated using the summation of the Millikan and White correlation ${ }^{23}$ with Park's high-temperature correction. ${ }^{24}$ Equations 14 and 15 respectively show the relaxation times using Millikan and White correlation and Park's high temperature correction, where $p$ is the pressure, $\sigma_{s}$ is the reference species collision cross section, $n$ is the total number density, and $m_{s}$ is the species mass. For this work, a reference collision cross section of $5.81 \times 10^{-21} \mathrm{~m}^{2}$ is used.

$$
\begin{gathered}
S_{v i b, s}^{t r a i b}=\rho_{s} \frac{e_{v i b, s}^{*}-e_{v i b, s}}{\tau_{v i b}} \\
\tau_{v i b, s}^{C F D}=\tau_{M W}+\tau_{P} \\
\tau_{M W}=\frac{1}{p} \exp \left[A_{s}\left(T_{t r a}^{-\frac{1}{3}}-B_{s}\right)-18.42\right] \\
\tau_{P}=\frac{\sqrt{\frac{\pi m_{s}}{8 k T_{t r a}}}}{\sigma_{s} n}
\end{gathered}
$$

The DSMC module has two different models available to simulate the vibration-translation relaxation process. The first model is a phenomenological model based on the method described by Boyd ${ }^{25}$ that calculates a variable probability of a vibrationally inelastic collision occurring based on the relative velocity of the two colliding particles. First, separate variable probabilities for the Millikan and White relaxation time and the Park high-temperature time are formed. The form of the Millikan and White variable probability is given by Eq. 16 where $Z_{o}, \alpha$ and $g^{*}$ are found from VHS parameters and by evaluating Eq. 17 such that the relaxation process reproduces macroscopic Millikan and White relaxation times under continuum conditions. Unfortunately, there are no methods to exactly calculate the integral in Eq. 17, so an approximation using the method of steepest descent is used. In a similar manner, an instantaneous probability can be formulated for Park's high temperature correction such that it satisfies Eq. 18. The total probability for a vibrationally inelastic collision can be formulated from the two variable probabilities as shown in Eq. 19. In order to account for the differences in the definition of relaxation times between DSMC and CFD, the Lumpkin correction factor shown in Eq. 9 is again applied to all DSMC vibrational relaxation times.

$$
\begin{gathered}
\phi_{M W}(g)=\frac{1}{Z_{o}} g^{\alpha} \exp \left(\frac{-g^{*}}{g}\right) \\
\frac{1}{\tau_{M W} \nu_{s}}=\int_{0}^{\infty} \phi_{M W}(g) f(g) d g \\
\frac{1}{\tau_{P} \nu_{s}}=\int_{0}^{\infty} \phi_{P}(g) f(g) d g \\
\phi_{\text {tot }}=\frac{\phi_{M W} \phi_{P}}{\phi_{M W}+\phi_{P}}
\end{gathered}
$$

Previous research has found that discrepancies in relaxation times between CFD and DSMC in areas that can be considered continuum have been attributed to approximating the number of vibrational degrees of freedom used in the Lumpkin correction factor and approximating the integral in Eq. 17 to evaluate the constants needed for the variable vibrational probability applied in DSMC simulations. Differences in the prediction of vibrational temperature contours between previous hybrid and full DSMC results have been partially attributed to differences in vibrational relaxation rates. ${ }^{14}$ In order to eliminate the contribution from the relaxation evaluation procedure, another method for calculating the probability of a vibrationally inelastic collision in DSMC is by directly evaluating the relaxation time in each cell and applying a constant probability of vibration-translation relaxation for each collision class over the entire cell. This method uses the macroscopic translational temperature to calculate the continuum relaxation time using Eq. 13 and applying the Lumpkin correction factor to find the DSMC relaxation time. The average probability within the cell 
is then calculated using Eq. 20 and applied to all collisions in the cell. Figure 1(b) shows a comparison of an adiabatic vibration relaxation process using the two DSMC methods and continuum calculation. Notice, that the cell based vibrational relaxation rate is in much better agreement with the continuum calculation than the variable vibrational relaxation probability. The effect of the improved agreement in prediction of vibrational relaxation between DSMC and CFD methods in continuum regions will be tested within a hybrid DSMC-CFD code in the proceeding sections.

$$
\bar{P}_{v i b}=\frac{1}{\tau_{v i b, s}^{D S M C} \nu_{s}}
$$

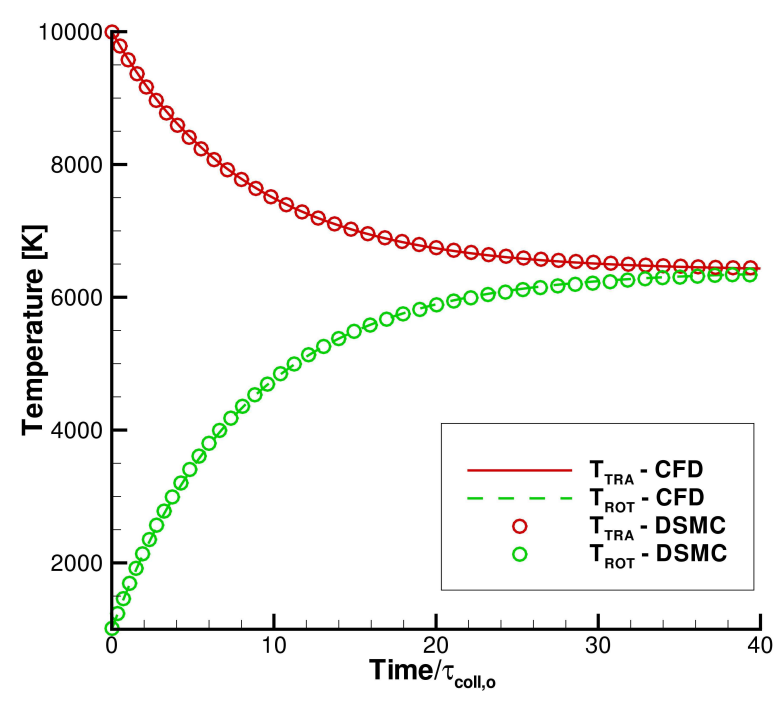

(a) Rotational Relaxation Process

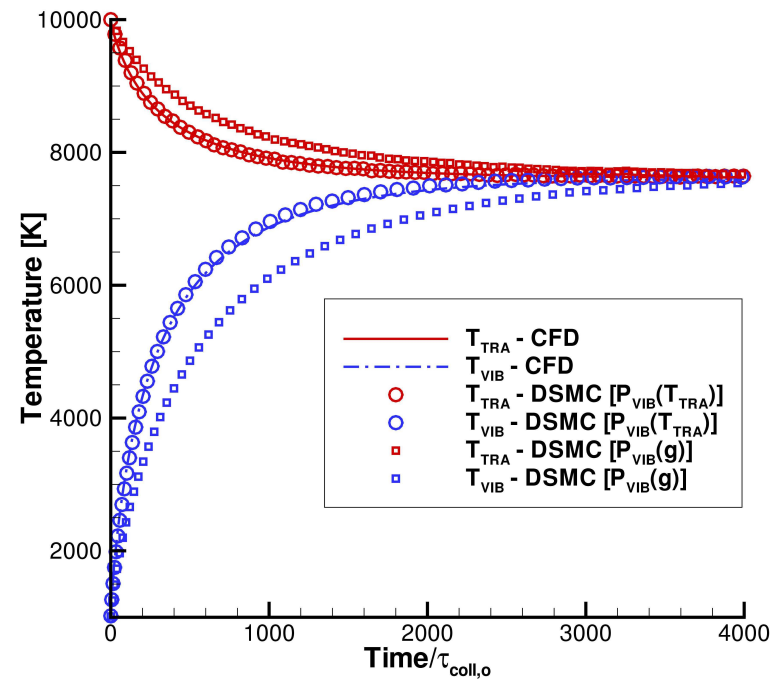

(b) Vibrational Relaxation Process

Figure 1. Comparison of adiabatic internal-translational relaxation processes predicted by DSMC and CFD.

\section{Hybrid Method}

The modular particle-continuum (MPC) method under development uses separate DSMC and CFD codes as modules within a hybrid framework. Very few modifications are made to each source code which allows the use of state of the art simulation methods that have been previously developed and verified. Instead the focus is on the hybrid methodology, particularly where the interface between the CFD and DSMC is located and when and how information should be transferred between the two modules.

\section{III.A. Interface Location and Continuum Breakdown}

Both the accuracy and efficiency of a hybrid DSMC-CFD method depend strongly on proper placement of the interface location. For physical accuracy, the interface location must be located within regions that can be considered in collisional equilibrium, where the velocity distribution is only slightly perturbed from equilibrium, and the Navier-Stokes equations are valid. The efficiency of the hybrid method requires the interface between CFD and DSMC to be located near the edge of the collisional equilibrium region. The MPC method uses a gradient-length Knudsen number first proposed by Boyd et al. ${ }^{26,27}$ Equation 21 shows the gradient-length Knudsen number where $\lambda$ is the local mean free path and $Q$ is some flow quantity of interest. For this work, gradient-length Knudsen numbers based on density, velocity magnitude, translational temperature, and rotational temperature are used. 


$$
K n_{G L-Q}=\lambda\left|\frac{\nabla Q}{Q}\right|
$$

Previous research has shown for hypersonic reentry problems ${ }^{11,28}$ and 1-D normal shock waves ${ }^{10}$ that regions where the maximum gradient-length Knudsen number remains less than 0.05 display less than a $5 \%$ difference in flow quantities predicted by DSMC and CFD assuming perfect gas physics. Therefore, regions where the maximum gradient-length Knudsen number exceeds 0.05 must be simulated with DSMC to maintain physical accuracy within the solution. Figure 2(a) shows a comparison of translational and rotational temperatures predicted by CFD and DSMC along the stagnation line of flow about a cylinder with a global Knudsen number of 0.01 and a free stream Mach number of 10 which were calculated in the work of Ref. 20. In addition, the location of collisional equilibrium breakdown predicted by the gradient-length Knudsen number is included. Notice the discrepancy between CFD and DSMC at the location of continuum breakdown is still significant. When only this breakdown parameter is used within the MPC method, the post-shock equilibrium temperature predicted in the CFD region of the MPC method is higher than that predicted by full DSMC which results in an over prediction in heat transfer to the body. To provide an adequately restrictive breakdown parameter near the body, this work uses an additional thermal parameter to limit the CFD domain in compression regions to areas where the rotational nonequilibrium is less than $10 \%$. This is performed by including a breakdown parameter based on rotational nonequilibrium given in Eq. 22. Figure 2(b) shows the interface location using the additional breakdown parameter. Now, the entire rarefied region along the stagnation streamline is simulated using the DSMC module and excellent agreement between full DSMC and the MPC method in the high compression region is possible. The proceeding results will show that further development of a breakdown parameter due to thermal nonequilibrium effects could improve agreement between the MPC method and full DSMC in regions located further from the body. Equation 23 shows the final breakdown parameter used by the MPC method which is the maximum of all breakdown parameters described.

$$
\begin{gathered}
K n_{R O T-N E Q}=\frac{1}{2} \times \frac{T_{T R A}-T_{R O T}}{T_{T R A}} \\
K n=\max \left(K n_{G L-\rho}, K n_{G L-T_{T R A}}, K n_{G L-T_{R O T}}, K n_{G L-|V|}, K n_{R O T-N E Q}\right)
\end{gathered}
$$

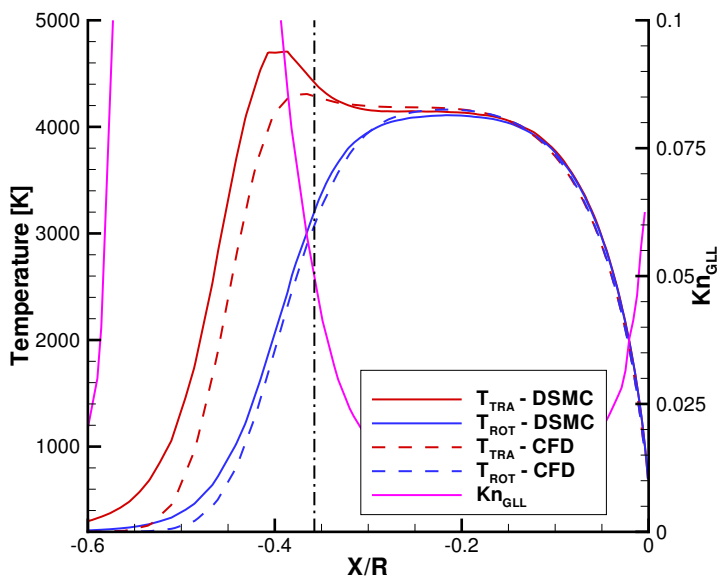

(a) Breakdown based on $K n_{G L L}$

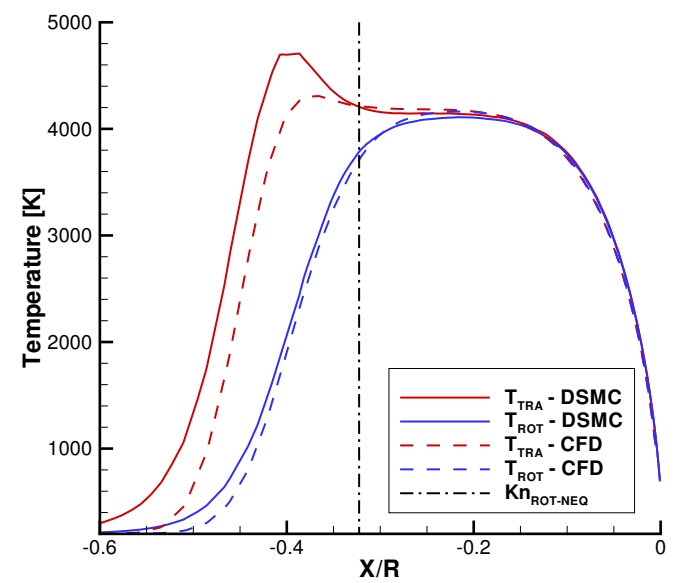

(b) Breakdown based on $K n_{R O T-N E Q}$

Figure 2. Comparison of temperatures between full DSMC and CFD and two continuum breakdown parameters along the stagnation streamline of a Mach 10 cylinder flow with global Knudsen number of 0.01 . 


\section{III.B. Information Transfer}

The modular implementation of the MPC method allows both the DSMC and CFD modules to maintain their own mesh and data structure. Information is transferred between each method using a state-based coupling procedure. The state-based coupling procedure assigns boundary cells on the edge of each module domain and transfers flow information, such as density, velocity components, and temperatures to these cells from corresponding cells of the other module. Figure 3 shows a schematic of how data is transferred between the two modules at an interface location.

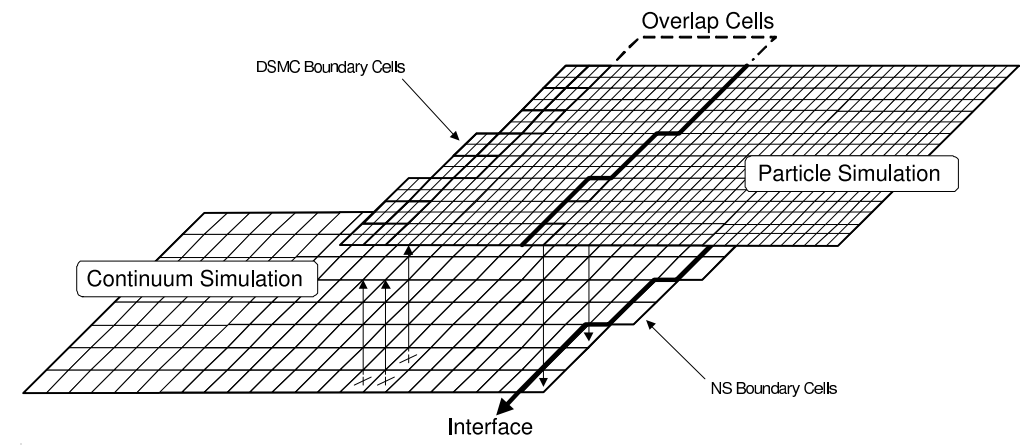

Figure 3. Hybrid particle-continuum coupling procedure. ${ }^{13}$

First, the interface location is found using the breakdown parameter shown in Eq. 23. Next, a DSMC buffer region is created. This buffer region is used throughout the unsteady portion of the simulation as the initial CFD solution relaxes to DSMC result. In order to obtain an accurate DSMC prediction in rarefied regions, the buffer region must be large enough to eliminate any error caused by the initially inaccurate DSMC boundary conditions from the CFD module. For this work, the buffer region is at least 20 mean free paths which corresponds to about $10 \mathrm{CFD}$ cells and ensures that a sufficient number of collisions occur to eliminate any error from the initial CFD solution propagating into the regions considered rarefied. Periodically, the breakdown parameter is re-evaluated to ensure that the entire rarefied region is contained within the DSMC domain. Once the interface location has stopped moving, information from the DSMC region is passed to the CFD edges and the CFD module iterates. Equation 24 shows the subrelaxation average that the MPC method uses to reduce the scatter in information collected from DSMC. ${ }^{29}$ This performs a weighted average that applies a small weight to the current sample while applying a larger weight to the previous average. The MPC method then periodically switches between the two modules, while allowing information to be transfered at each module switch as the entire flow field iterates to the steady-state solution.

$$
\bar{Q}_{j}=(1-\Phi) \bar{Q}_{j-1}+\Phi Q_{j}
$$

Figure 4 shows the relative position of the shock location as the MPC method iterates to steady state compared to the shock location predicted by DSMC for Mach 15 flow over a cylinder with a global Knudsen number of 0.01. At first, the shock is located closer to the body which agrees with the initial full CFD solution. Then as the DSMC region iterates, the shock moves out past the full DSMC prediction which is due to the initially inaccurate boundary condition from CFD in the post-shock region. Once the interfaces stop moving, coupling between the two modules occurs, and the shock moves to less than $1 \%$ of the location predicted by DSMC.

\section{Flow Conditions}

Hypersonic flow about a cylinder at Mach 15 is simulated. The free-stream gas simulated is $N_{2}$ with a free stream number density of $n_{\infty}=1.61 \times 10^{21} \mathrm{~m}^{-3}$ and equilibrium temperatures set at $T_{\text {tra, } \infty}=T_{\text {rot }, \infty}=$ $T_{v i b, \infty}=217.5 \mathrm{~K}$. This corresponds to a free stream density of $\rho_{\infty}=7.48 \times 10^{-5} \mathrm{~kg} \mathrm{~m}^{-3}$ and a pressure of $p_{\infty}=4.22 \mathrm{~Pa}$. The diameter of the cylinder simulated is $d=8 \mathrm{~cm}$ which results in a global Knudsen number of $K n_{\infty}=0.01$. The cylinder wall temperature is set to $T_{w}=1,000 \mathrm{~K}$ with full diffuse reflection in DSMC simulations and a no-slip, isothermal condition in CFD simulations. The vibrational characteristic temperature used for all vibration temperature calculations is $\theta_{v i b}=3390 \mathrm{~K}$. A constant time-step of 


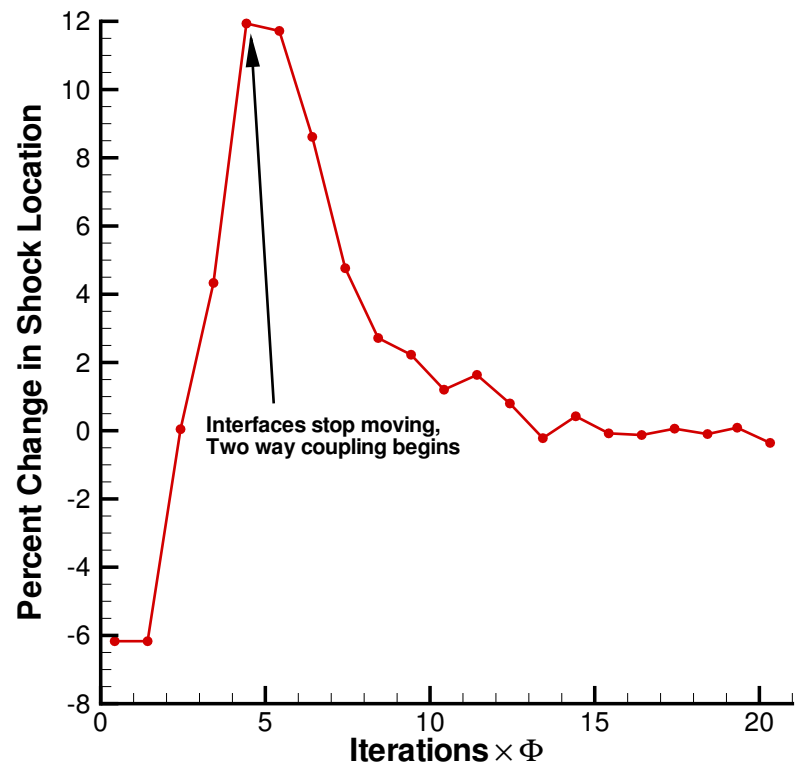

Figure 4. Progression of the shock location during the unsteady portion of the hybrid coupling cycle

$1.5 \times 10^{-8} s$ is used in the DSMC method for all simulations, while a maximum CFL number of 50 is used in all CFD simulations.

Results with different physical models are compared. Case M15 3 T-VP corresponds to the case where full thermal (vibrational and rotational) nonequilibrium are simulated in both modules and vibrational relaxation in the DSMC module is simulated using the variable probability method based on the relative velocity. Case M15 $_{3 \mathrm{~T}-\mathrm{CB}}$ corresponds to the case where full thermal nonequilibrium is simulated within both modules and vibrational relaxation in the DSMC module is simulated using the cell-based constant probability method. Finally, a third case, $\mathbf{M} 15_{\mathrm{NV}}$, is computed where vibrational excitation is ignored in both modules to assess agreement of rotational nonequilibrium between DSMC and the MPC method.

\section{Numerical Results of Nonequilibrium Flows}

The purpose of the MPC method is to reproduce full DSMC results to within $5 \%$ at a reduced computational expense. This section compares flow field and surface property results obtained with full DSMC, full CFD, and the MPC method for each of the flow conditions. In addition, code efficiency of the MPC method compared to full DSMC is discussed.

\section{V.A. Flow Field Properties}

Figure 5 shows the final and initial interface locations for the $\mathbf{M} 15_{\mathrm{NV}}$ case. The interface locations for all three cases are very similar, so only one is shown. Also, the two extraction lines that are used to compare flow variables are indicated.

Figure 6(a) compares the translational temperature contours calculated using full DSMC, full CFD, and the MPC method for the $\mathbf{M 1 5}_{\mathrm{NV}}$ case. Overall, the MPC method is able to reproduce full DSMC results while limiting the DSMC computation to areas that are in collisional nonequilibrium. The largest discrepancy between full DSMC and the MPC results is below 4\%. This occurs far away from the body behind the shock, near the $4,000 \mathrm{~K}$ contour level. This discrepancy is thought to be caused by continuum breakdown due to strong rotation-translation nonequilibrium effects. Decreasing the rotation-translation nonequilibrium tolerance for the CFD module reduces the discrepancy far away from the body, but at the expense of unnecessarily increasing the DSMC domain in the forebody region. Instead, a different breakdown parameter that takes into account nonequilibrium thermal relaxation could provide a better estimation of 


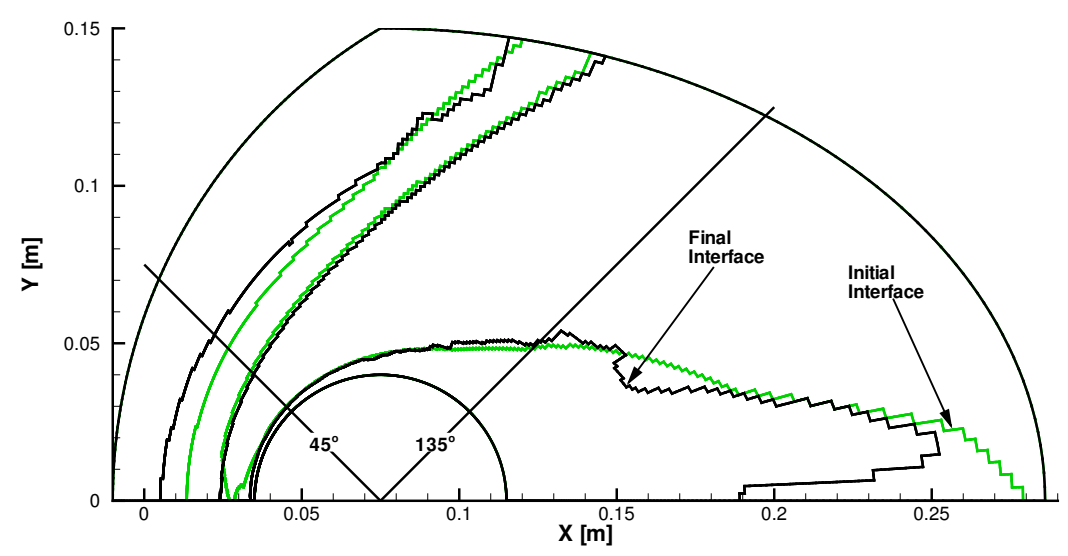

Figure 5. Initial and final DSMC-CFD interface locations for the M15 $\mathrm{M}_{3}$-CB case. Also, extraction lines used to compare flow fleld data are shown.

the onset of collisional nonequilibrium. Figure 6(b) compares the rotational temperature contours predicted by DSMC, CFD, and the MPC method. Again, agreement is very good with the largest differences of less than $3 \%$ occurring far away from the body.

Figure 7(a) shows the prediction of flow field variables by CFD, DSMC, and the MPC method for the M15 $\mathrm{NV}$ case along the $45^{\circ}$ cut labeled in Fig. 5. There is excellent agreement between full DSMC and the MPC method along the entire extraction line. Even in regions where the CFD module is used, the MPC method improves the agreement with full DSMC compared to the initial full CFD solution. Figure 7(b) shows the comparison of flow variables along the $135^{\circ}$ extraction line labeled in Fig. 5. In general, full DSMC and CFD are in close agreement along most of the extraction line and the MPC method maintains this same level of agreement. However, near the body, full DSMC and CFD are not in good agreement, while the MPC solution remains in excellent agreement with the full DSMC solution. This improvement near the surface provides a direct improvement on the calculated heat transfer to the body compared with full DSMC.

Next, Figs. 8(a) and (b) compare the translational and vibrational temperature contours predicted by DSMC, CFD, and the MPC method for the M15 $\mathbf{M T}_{\mathbf{3 T}-\mathrm{VP}}$ case. For the prediction of translational temperatures contours, the MPC method is in much better agreement with full DSMC than full CFD is with full DSMC. The largest discrepancy measured between the MPC and DSMC results for any flow variable other than the vibrational temperature remains below $4 \%$. When comparing vibrational temperature contours, the MPC method greatly improves agreement with the full DSMC results. The worst agreement occurs in the expansion region where the MPC and full DSMC predicted vibrational temperatures deviate up to about $8 \%$. This could partially be attributed to the difference in relaxation rates computed in full DSMC and the CFD module of the MPC in this region.

Figure 9(a) shows a comparison of translational temperature contours predicted by full DSMC, full CFD, and the MPC method for the M15 $\mathbf{5}_{\mathbf{3 T}-\mathrm{CB}}$ case. Again, agreement between DSMC and the MPC predictions is excellent while full CFD still fails to accurately predict nonequilibrium flow field features. The largest discrepancy found between DSMC and the MPC results remains below $5 \%$ for all flow variables. Figure 9(b) compares the vibrational temperature contours predicted by full DSMC, CFD, and the MPC method. Again, the MPC method can accurately reproduce the full DSMC solution even in areas where full DSMC and full CFD are in very poor agreement. Still, there is a discrepancy between the MPC method and full DSMC in the expansion region, but the agreement between full DSMC and the MPC method has improved with the maximum difference between DSMC and the MPC result remaining below $4 \%$. This is directly due to the improved agreement in the modeling of vibrational relaxation rates in continuum regions.

Figures 10(a) and (b) show the vibrational temperature predicted by DSMC, CFD, and the MPC method along the $135^{\circ}$ extraction line for the $\mathbf{M 1 5}_{3 \mathrm{~T}-\mathrm{VP}}$ and $\mathbf{M} 15_{3 \mathrm{~T}-\mathrm{CB}}$ cases, respectively. As was shown in the vibrational temperature contour plots, the MPC method greatly improves agreement with full DSMC compared to full CFD. Despite the improved agreement, the effect of different relaxation rates in the M15 $5_{3 T-V P}$ 


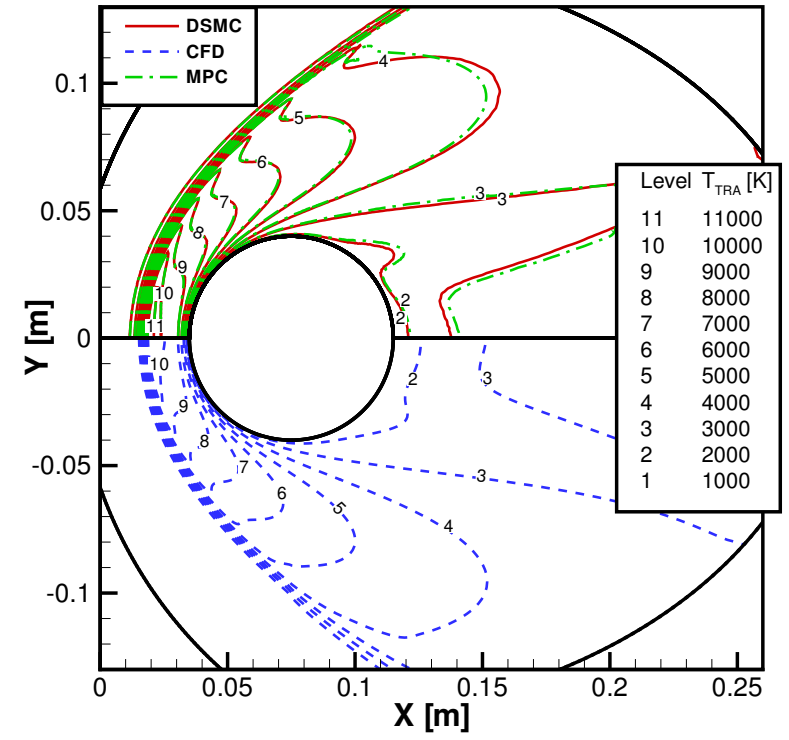

(a) $T_{\text {tra }}$

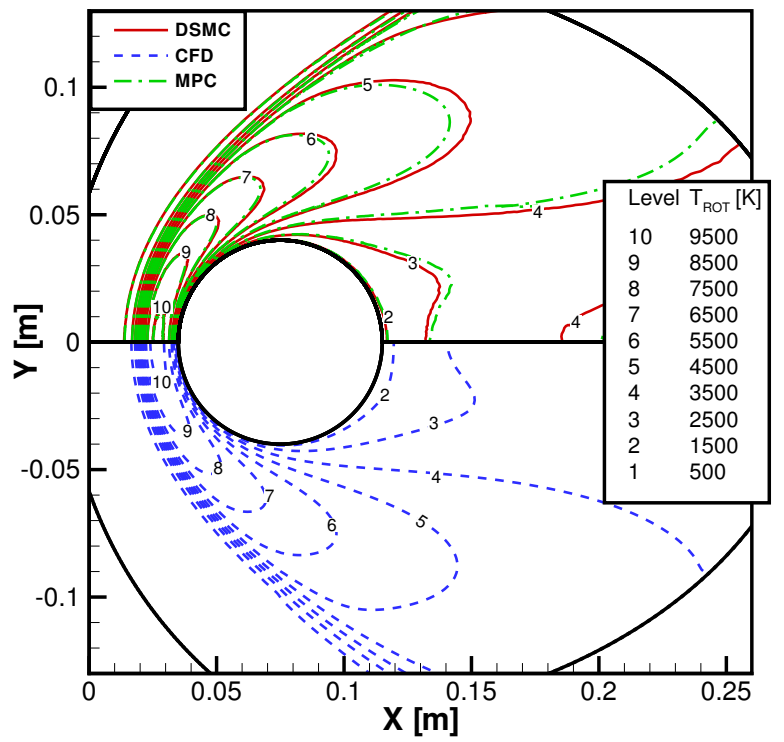

(b) $T_{\text {rot }}$

Figure 6. Comparison of temperature contours predicted by DSMC, CFD, and the MPC method for the M15 ${ }_{\mathrm{NV}}$ case.

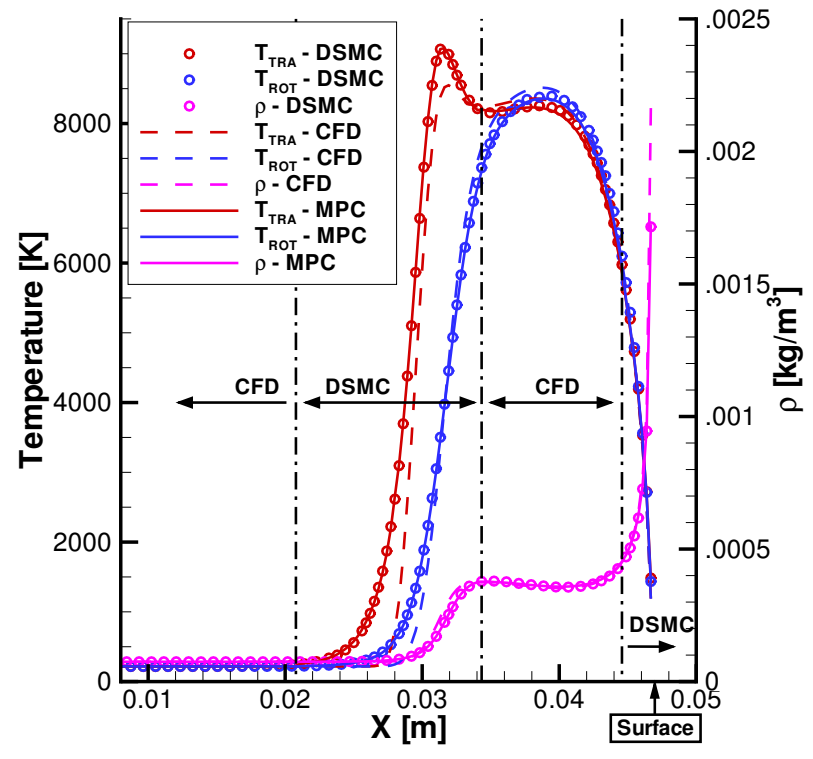

(a) $45^{\circ}$ extraction line.

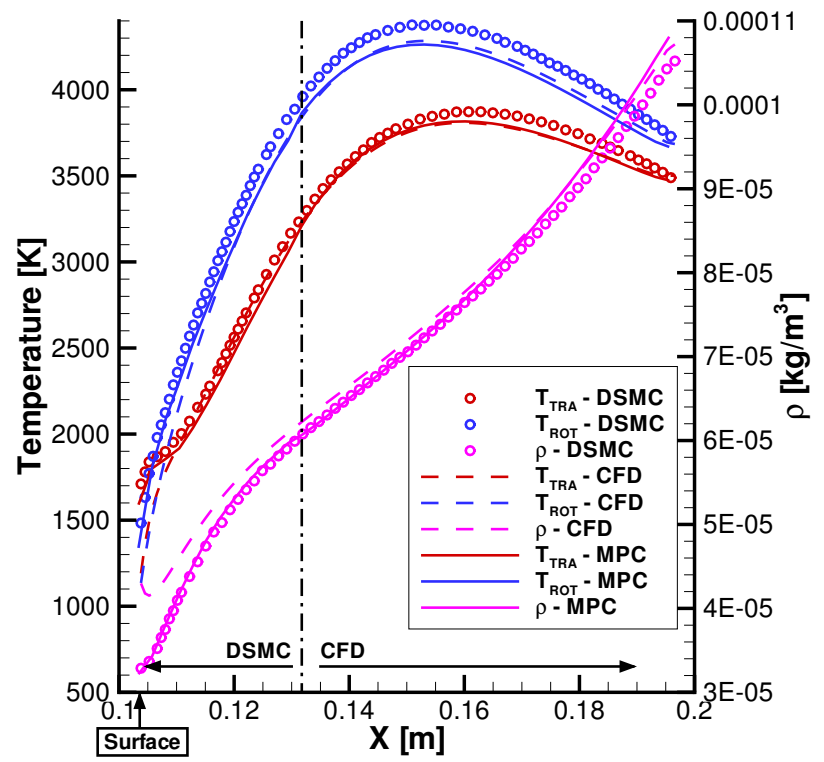

(b) $135^{\circ}$ extraction line.

Figure 7. Comparison along extraction lines of temperatures and density predicted by DSMC, CFD, and the MPC method for the M15 NV case. 


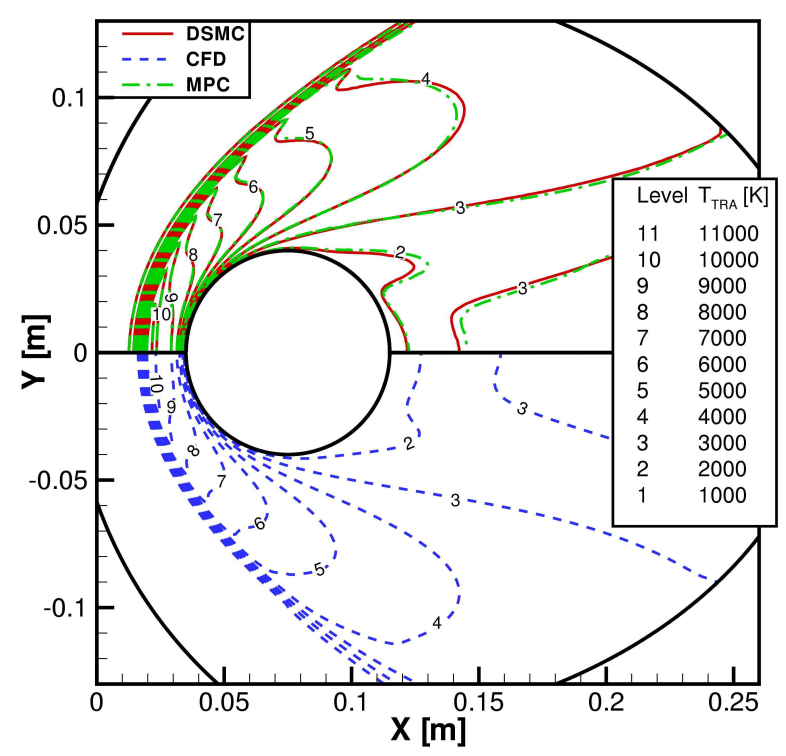

(a) $T_{T R A}$

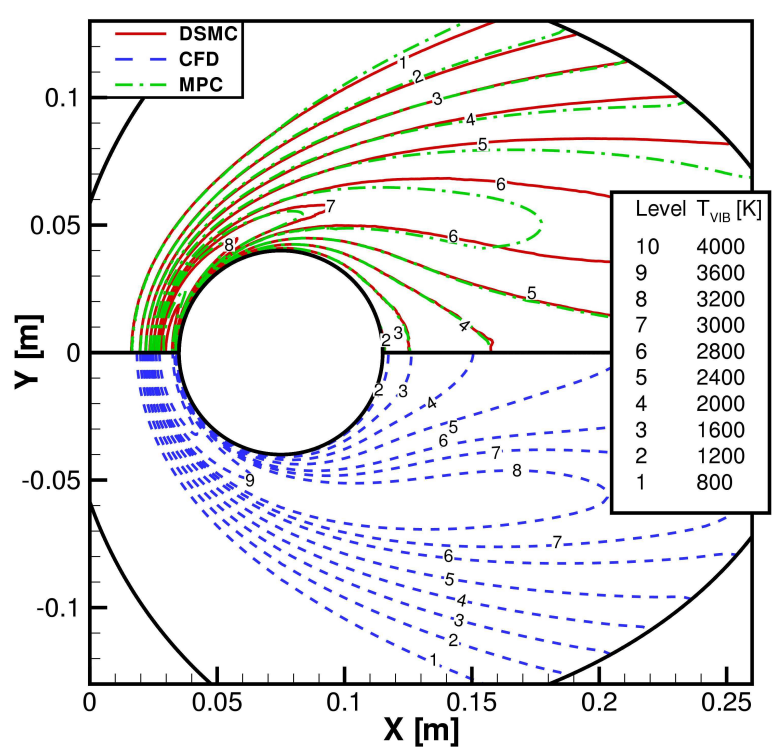

(b) $T_{V I B}$

Figure 8. Comparison of temperature contours predicted by DSMC, CFD, and the MPC method for the M15 ${ }_{3 T-V P}$ case.

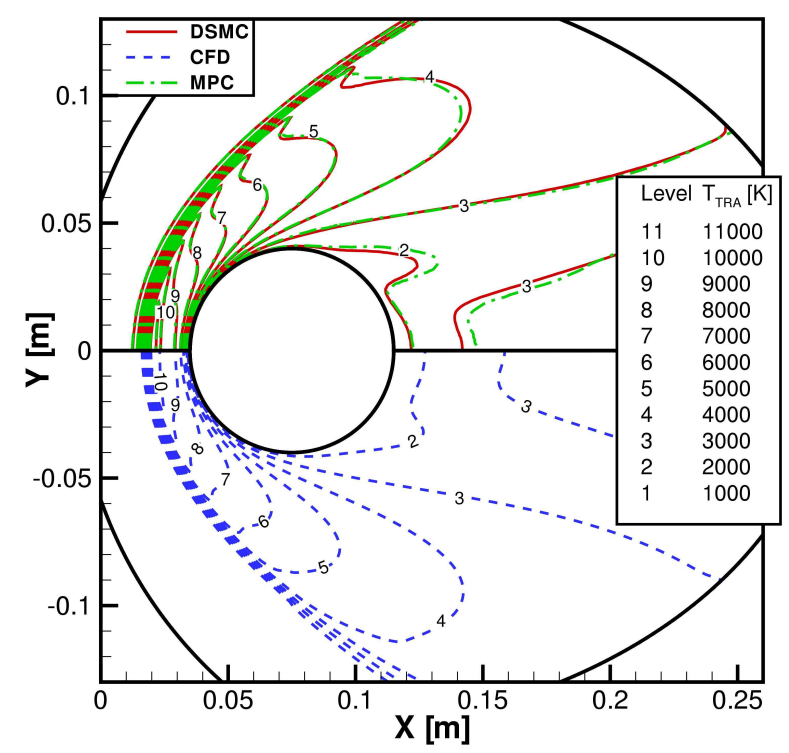

(a) $T_{T R A}$

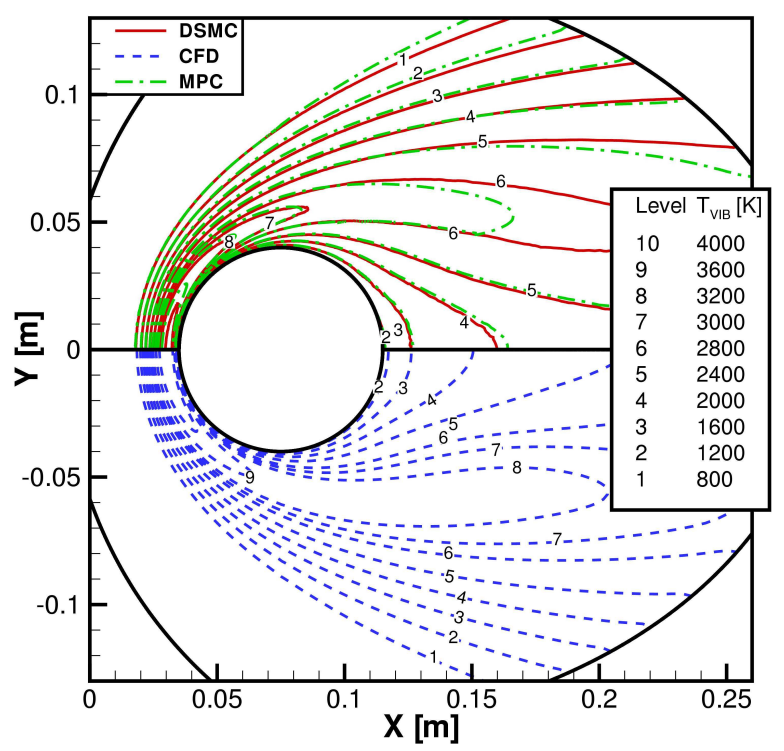

(b) $T_{V I B}$

Figure 9. Comparison of temperature contours predicted by DSMC, CFD, and the MPC method for the M15 $5_{3 T-C B}$ case. 
is evident at the interface location between the CFD and DSMC modules. There is a distinct change in vibrational temperature gradient. This is caused by the difference in relaxation rates computed in each module. When compatible relaxation rates are used in the two modules, as is done for the $\mathbf{M 1 5 _ { 3 T - C B }}$ case, the solution is smooth with no abrupt change in gradient at the interface. Though small for this test case, differences in relaxation rates could have a larger effect on agreement between full DSMC and the MPC method for flows where the continuum region exhibits faster relaxation rates that would occur in higher enthalpy flows.

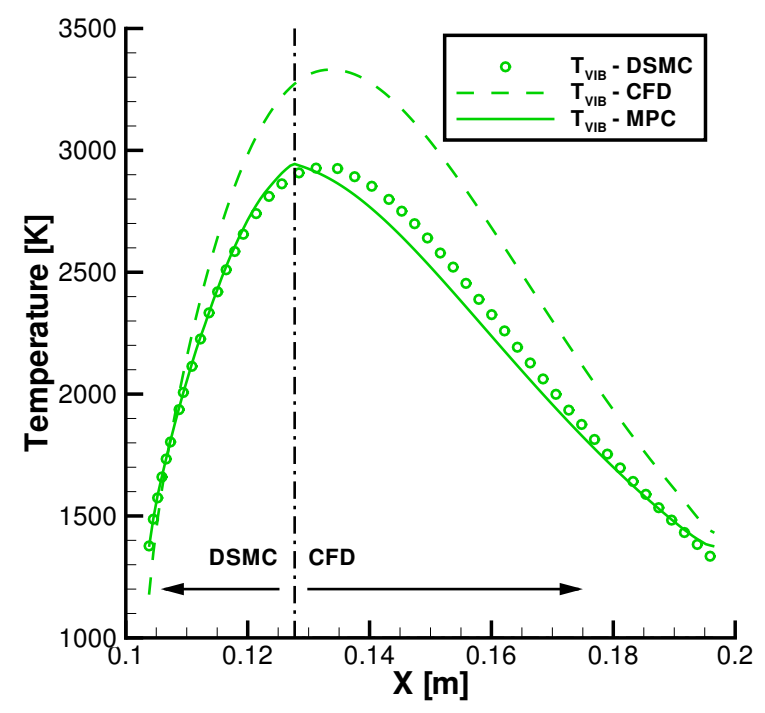

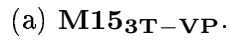

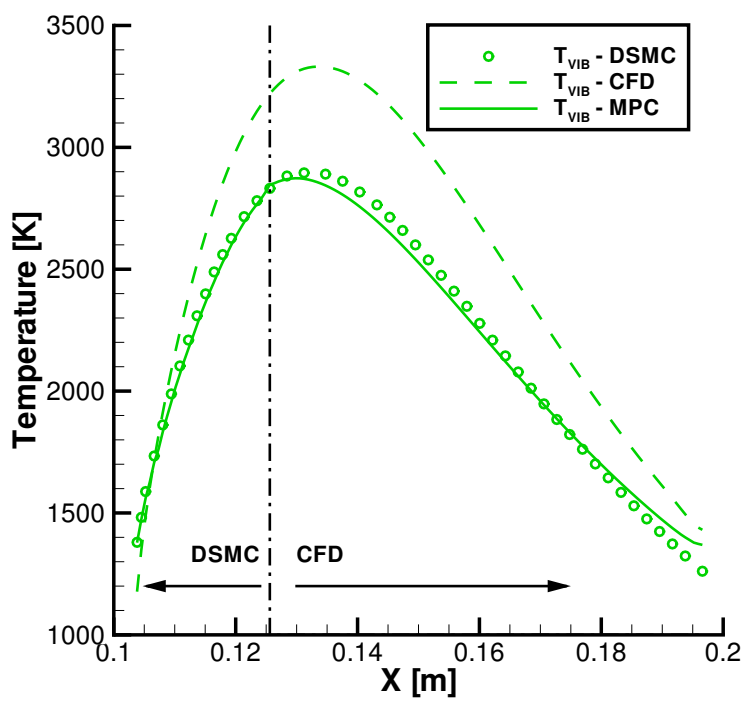

(b) $\mathrm{M}^{15_{3 \mathrm{~T}}-\mathrm{CB}}$.

Figure 10. Comparison of vibrational temperature predicted by DSMC, CFD, and the MPC method along the $135^{\circ}$ extraction line for the $\mathrm{M15}_{3 \mathrm{~T}-\mathrm{VP}}$ and $\mathrm{M} 15_{3 \mathrm{~T}-\mathrm{CB}}$ cases.

\section{V.B. Surface Properties}

For many planetary entry problems of interest, accurate prediction of surface properties has a strong impact on vehicle design. Predictions of heat transfer and pressure from full DSMC, full CFD, and the MPC method are compared. Equations 25 and 26 respectively show the definitions of the heat transfer coefficient and surface pressure coefficient used to compare the surface properties predicted by the simulation methods where $q$ is the heat transfer to the wall, $p$ is the pressure at the wall, $p_{\infty}$ is the free stream pressure, $\rho_{\infty}$ is the free stream density, and $V_{\infty}$ is the free stream velocity.

$$
\begin{aligned}
C_{h} & =\frac{q}{\frac{1}{2} \rho_{\infty} V_{\infty}^{3}} \\
C_{p} & =\frac{p-p_{\infty}}{\frac{1}{2} \rho_{\infty} V_{\infty}^{2}}
\end{aligned}
$$

Figure 11(a) compares the heat transfer coefficient to the cylinder surface predicted by DSMC, CFD, and the MPC method for the $\mathbf{M 1 5}_{\mathrm{NV}}$ case. All three methods remain within $5 \%$ of each other in the forebody region while the full CFD solution deviates from the full DSMC and MPC results as the flow expands around the cylinder. The largest difference between DSMC and CFD is located in the afterbody of the cylinder where CFD over predicts the heat transfer to the surface by up to a factor of 3 . In contrast, the MPC method remains in excellent agreement with DSMC along the entire cylinder body. Figure 11(b) compares 
the coefficient of pressure along the surface predicted by DSMC, CFD, and the MPC method. In general the results predicted by the three methods are in agreement along the fore body of the cylinder. CFD again over predicts the DSMC results by up to $50 \%$ in the wake region, while the MPC results are in excellent agreement with DSMC.

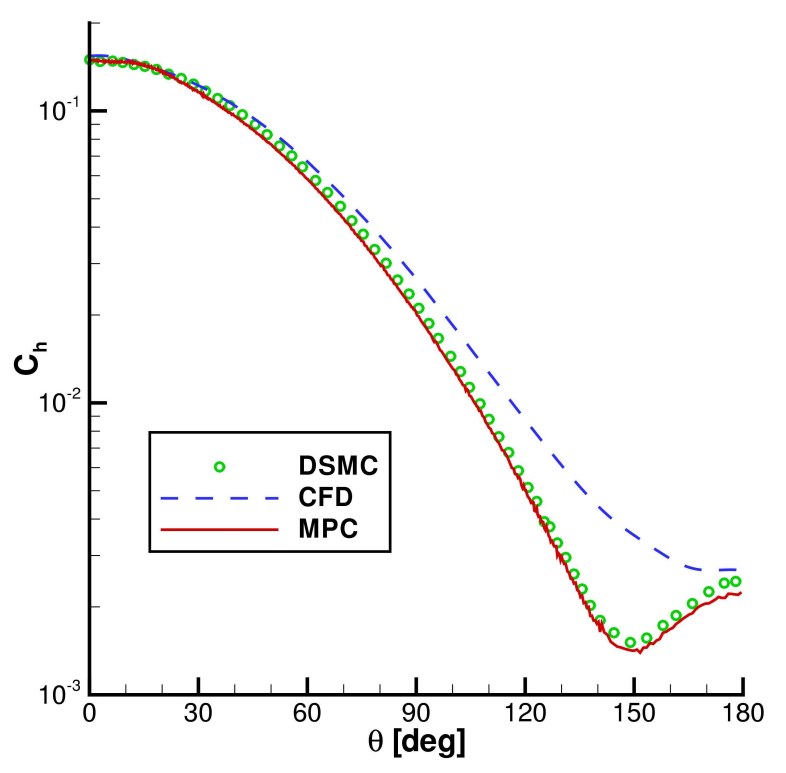

(a) $C_{h}$

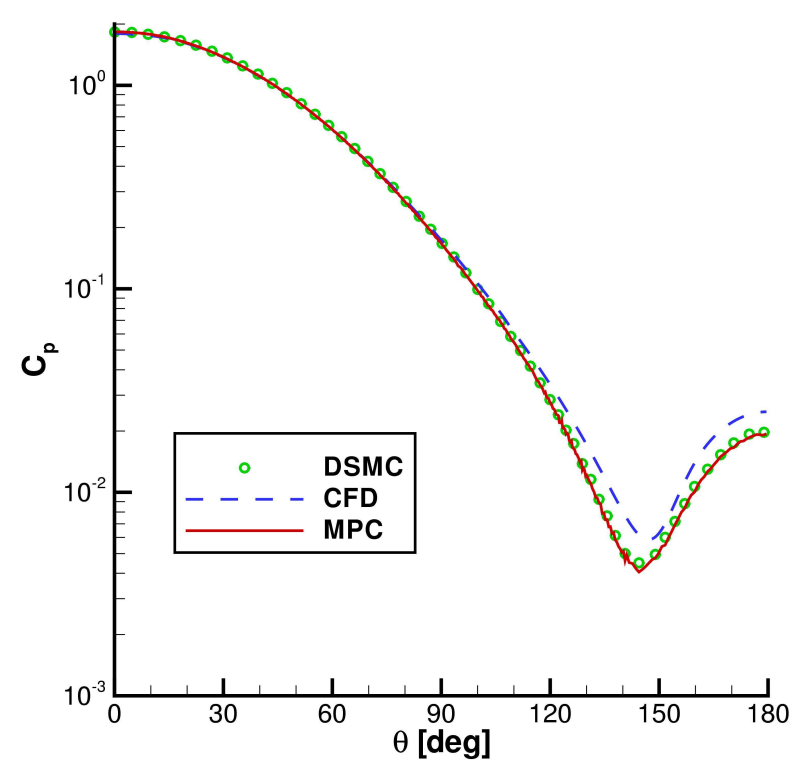

(b) $C_{p}$

Figure 11. Comparison of heat transfer and pressure coefficient along the cylinder surface predicted by DSMC, CFD, and the MPC method for the M15 $\mathrm{NV}$ case.

Figures 12(a) and (b) show the heat transfer coefficient predicted by DSMC, CFD, and the MPC method for the $\mathbf{M 1 5}_{3 \mathrm{~T}-\mathrm{VP}}$ and $\mathbf{M 1 5}_{3 \mathrm{~T}-\mathrm{CB}}$ cases, respectively. Despite the better agreement in the prediction of vibrational temperature between DSMC and the MPC method for the M15 $\mathbf{5}_{3 \mathrm{~T}-\mathrm{CB}}$, both cases have the same level of agreement between the MPC method and full DSMC in heat transfer to the body. This is due to the MPC results being in excellent agreement with full DSMC near the body for both cases. Again the MPC method is able to reproduce full DSMC results, especially in the wake region where CFD over predicts the heat transfer to the body by a factor of 3 .

\section{V.C. Computational Performance}

The MPC method reproduces full DSMC results by using DSMC in rarefied regions while decreasing the computational cost by limiting DSMC to only regions that are in collisional nonequilibrium. Continuum regions are computed using an implicit Navier-Stokes solver. By loosely coupling the methods and studying steady-state flows, the time-step used by CFD can be over 100 times larger than the time-step required by DSMC for the corresponding flow. In addition, decoupling of the mesh densities allows CFD to be unrestricted by DSMC cell size restrictions, and can use cells that are up to 18 times larger in area. This significantly decreases the computational time and memory usage required by the MPC method to reproduce full DSMC results. The decreases in computational cost of the MPC method compared to full DSMC are summarized in Table 1. To make a fair comparison, the number of sample time-steps after reaching steadystate are the same for both full DSMC and the MPC DSMC module. Here, the actual speedup is defined as the ratio of the time required for the full DSMC calculation to the time required for the MPC method calculation. The ideal speedup is defined as the ratio of particles used in a full DSMC simulation compared to the number in the corresponding MPC simulation. Since the computational cost of a DSMC simulation nearly scales linearly with the number of particles in the simulation, this should be the upper limit for 


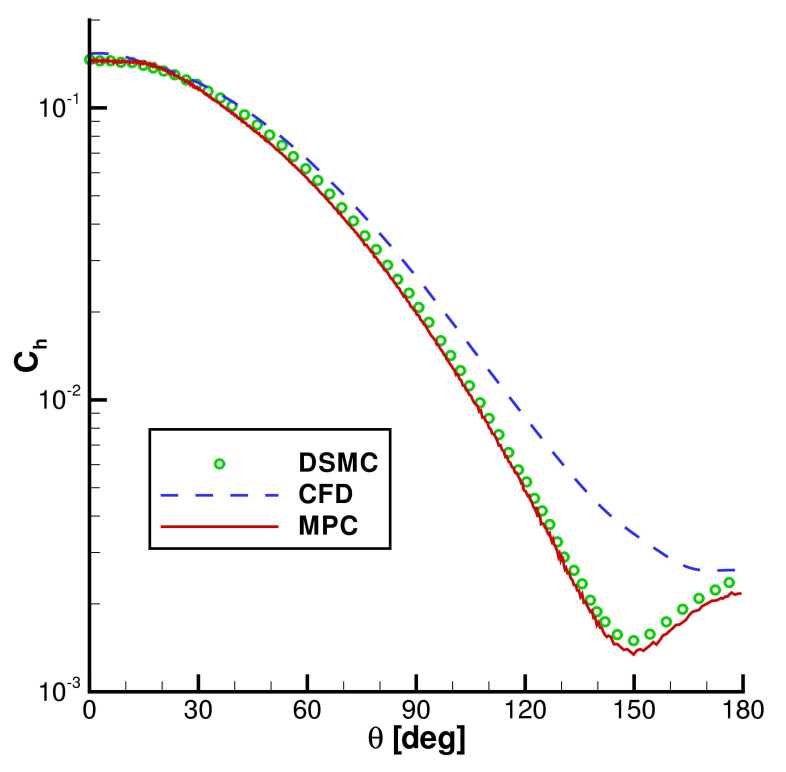

(a) $\mathrm{M15}_{3 \mathrm{~T}}-\mathrm{VP}$.

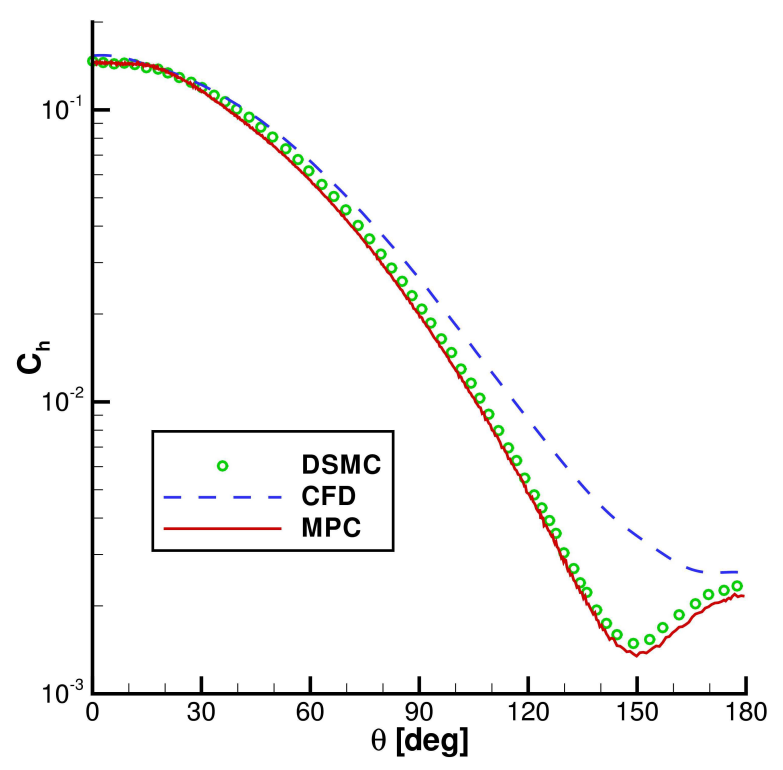

(b) $\mathrm{M15}_{3 \mathrm{~T}-\mathrm{CB}}$

Figure 12. Comparison of coefficient of heat transfer along the cylinder surface predicted by DSMC, CFD, and the MPC method for the $\mathrm{M15}_{3 \mathrm{~T}-\mathrm{VP}}$ and $\mathrm{M} 15_{3 \mathrm{~T}-\mathrm{CB}}$ cases.

computational speedup for that problem. The MPC method actually outperforms the ideal speedup ratio. This is because the unsteady portion of the MPC computation is much less than that of the corresponding DSMC simulation since MPC simulations begin with a fully converged CFD solution. In addition, the cost of the MPC method for the $\mathbf{M 1 5}_{\mathrm{NV}}$ case is much less than the cost of the other two cases which include vibrational nonequilibrium. This is because the computational cost of the CFD module increases dramatically with the inclusion of vibrational nonequilibrium due to the nonlinear vibrational temperatureenergy relation. Since vibrational temperature can not be found analytically in a general multi-species flow, an iterative scheme must be used to solve for the vibrational temperature which further increases the computational cost of including vibrational nonequilibrium within the CFD module. The memory usage is defined as the ratio of the memory used by an MPC simulation to the memory used by the corresponding DSMC simulation. Here, the MPC method requires significantly less memory compared to full DSMC.

Table 1. Computational performance and memory requirements for the MPC method.

\begin{tabular}{|c|c|c|c|}
\hline Case & Actual Speedup & Ideal Speedup & Memory Usage \\
\hline \hline $\mathbf{M 1 5}_{\mathbf{N V}}$ & 4.53 & 2.62 & $22 \%$ \\
\hline $\mathbf{M 1 5}_{\mathbf{3 T}-\mathbf{V P}}$ & 3.37 & 3.07 & $22 \%$ \\
\hline $\mathbf{M 1 5}_{\mathbf{3 T}-\mathbf{C B}}$ & 3.96 & 3.16 & $27 \%$ \\
\hline
\end{tabular}

\section{Conclusion}

The implementation of rotational relaxation within a modular particle-continuum (MPC) method was described and tested on a hypersonic, blunt-body flow where regions of the flow exhibited collisional nonequilibrium. An MPC method is outlined that uses existing DSMC and CFD codes with very little modification as modules within a hybrid code. This implementation allows separate updates of either the DSMC or CFD source codes which reduces the development time of the hybrid code as newer physical models are added 
to each code. Comparison of the effect of the vibrational relaxation model used in the DSMC module was performed. It was found that a slight improvement in agreement of vibrational temperatures was possible, but did not have an effect on surface properties. With the inclusion of added physical models, such as finite rate chemistry, these improvements could have a larger effect on surface properties.

In addition, it was found that the empirical gradient-length Knudsen number was not quite adequate to predict the onset of collisional nonequilibrium in the post-shock region. This could be due to the strong thermal nonequilibrium that occurs in the post shock region. For this work, a thermal nonequilibrium parameter was added. Although some recent work has been performed on formulating new breakdown parameters ${ }^{28}$ for use in hybrid CFD-DSMC methods, further research toward the development of an improved predictor of breakdown of collisional nonequilibrium may provide a more adequate breakdown parameter for higher enthalpy flows

Comparison of near-equilibrium flow fields predicted by full DSMC, full CFD, and the MPC method showed that by only using DSMC in regions that are are in collisional nonequilibrium, the MPC method was able to reproduce full DSMC results while reducing the computational expense.

\section{Acknowledgments}

The authors gratefully acknowledge the financial support provided by NASA grant NCC3-989. The second author acknowledges support under the NASA graduate student researchers program fellowship program (NNX07AV91H) through NASA Ames Research Center monitored by Dr. Michael Wright. The fourth author also acknowledges support from the Air Force Office of Scientific Research (AFOSR) under Grant No. FA9550-04-1-0341. The views and conclusions contained herein are those of the authors and should not be interpreted as necessarily representing the official policies or endorsements, either expressed or implied, of the AFOSR or the U.S. Government.

\section{References}

${ }^{1}$ Bird, G. A., Molecular Gas Dynamics and the Direct Simulation of Gas Flows, Clarendon Press, 1994.

${ }^{2}$ Glass, C. E. and Gnoffo, P. A., "A 3-D Coupled CFD-DSMC Solution Method With Application to the Mars Sample Return Orbiter," Tech. Rep. TM-2000-210322, NASA, July 2000.

${ }^{3}$ Glass, C. E. and Gnoffo, P. A., "Comparison of a 3-D CFD-DSMC Soultion Methodology With a Wind Tunnel Experiment," Tech. Rep. TM-2002-211777, NASA, August 2002.

${ }^{4}$ Wilmoth, R. G., Mitcheltree, R. A., Moss, J. N., and K., D. V., "Zonally Decoupled Direct Simulation Monte Carlo Solutions of Hypersonic Blunt-Body Flows," Journal of Spacecraft and Rockets, Vol. 31, No. 6, 1994, pp. 971-979.

${ }^{5}$ Hash, D. B. and Hassan, H. A., "Assessment of Schemes for Coupling Monte Carlo and Navier-Stokes Soultion Methods," Journal of Thermophysics and Heat Transfer, Vol. 10, No. 2, 1996, pp. 242-249.

${ }^{6}$ Hash, D. B. and Hassan, H. A., "A Decoupled DSMC/Navier-Stokes Analysis of a Transitional Flow Experiment," Collection of Technical Papers - 34th AIAA Aerospace Sciences Meeting and Exhibit, Vol. 1, Reno, NV, 1996.

${ }^{7}$ Roveda, R., B., G. D., and Varghese, P. L., "Hybrid Euler/Particle Approach for Continuum/Rarefied Flows," Journal of Spacecraft and Rockets, Vol. 35, No. 3, 1998, pp. 258-265.

${ }^{8}$ Roveda, R., B., G. D., and Varghese, P. L., "Hybrid Euler/Direct Simulation Monte Carlo of Unsteady Slit Flow," Journal of Spacecraft and Rockets, Vol. 37, No. 6, 2000, pp. 753-760.

${ }^{9}$ Wijesinghe, H. S. and G., H. N., "A discussion of Hybrid Atomistic-Continuum Methods for Multiscale Hydrodynamics," International Journal for Multiscale Computational Engineering, Vol. 2, 2004.

${ }^{10}$ Schwartzentruber, T. E. and Boyd, I. D., "A hybrid particle-continuum method applied to shock waves," Journal of Computational Physics, Vol. 215, No. 2, July 2006, pp. 402-416.

${ }^{11}$ Schwartzentruber, T. E., Scalabrin, L. C., and Boyd, I. D., "Hybrid particle-continuum simulations of nonequilibrium hypersonic blunt-body flowfields," Journal of Thermophysics and Heat Transfer, Vol. 22, No. 1, 2008, pp. $29-37$.

${ }^{12}$ Schwartzentruber, Thomas E.and Scalabrin, L. C. and Boyd, I. D., "Multiscale Particle-Continuum Simulations of Low Knudsen Number Hypersonic Flow Over a Planetary Probe," Journal of Spacecraft and Rockets, Vol. 45, No. 6, 2008, pp. 11961206.

${ }^{13}$ Schwartzentruber, T. E., Scalabrin, L. C., and Boyd, I. D., "Modular implementation of a hybrid DSMC-NS algorithm for hypersonic non-equilibrium flows," AIAA-2007-613.

${ }^{14}$ Deschenes, T. R., Boyd, I. D., and Schwartzentruber, T. E., "Incorporating Vibrational Excitation in a Hybrid ParticleContinuum Method," AIAA-2008-4106.

${ }^{15}$ Holman, T. D. and Boyd, I. D., "Numerical Investigation of the Effects of Continuum Breakdown on Hypersonic Vehicle Surface Properties," No. AIAA 2008-3928, 2008.

${ }^{16}$ Schwartzentruber, T. E., Scalabrin, L. C., and Boyd, I. D., "A modular particle-continuum numerical method for hypersonic non-equilibrium gas flows," Journal of Computational Physics, Vol. 225, No. 1, July 2007, pp. 1159-1174.

${ }^{17}$ Scalabrin, L. C. and Boyd, I. D., "Numerical simulation of weakly ionized hypersonic flow for reentry configurations," Vol. 4, San Francisco, CA, United States, AIAA-2006-3773, pp. 2310-2327. 
${ }^{18}$ Scalabrin, L. C. and Boyd, I. D., "Development of an Unstructured Navier-Stokes Solver for Hypersonic Nonequilibrium Aerothermodynamics," 38th AIAA Thermophysics Conference, Toronto, Canada, June 6-9 2005.

${ }^{19}$ Dietrich, S. and Boyd, I. D., "Scalar and Parallel Optimized Implementation of the Direct Simulation Monte Carlo Method," Journal of Computational Physics, Vol. 126, No. 2, July 1996, pp. 328-342.

${ }^{20}$ Holman, T. D. and Boyd, I. D., "Numerical Investigation of the Effects of Continuum Breakdown on Hypersonic Vehicle Surface Properties," AIAA Paper 2008-3928, Seattle, WA, United States, 2008.

${ }^{21}$ Parker, J. G., "Rotational and Vibrational Relaxation in Diatomic Gases," Phys. Fluids, Vol. 2, No. 4, July 1959, pp. 449-462.

${ }^{22}$ Boyd, I. D., "Analysis of rotational nonequilibrium in standing shock waves of nitrogen," AIAA Journal, Vol. 28, No. 11, 1990, pp. $1997-1999$.

${ }^{23}$ Millikan, R. C. and White, D. R., "Systematics of Vibrational Relaxation," The Journal of Chemical Physics, Vol. 39, No. 12, December 1963, pp. 3209-3213.

${ }^{24}$ Park, C., Nonequilibrium Hypersonic Aerothermodynamics, John Wiley \& Sons, 1990.

${ }^{25}$ Boyd, I. D., "Analysis of vibrational-translational energy transfer using the direct simulation Monte Carlo method," Phys. Fluids A, Vol. 3, No. 7, July 1991, pp. 1785-1791.

${ }^{26}$ Boyd, I. D., Chen, G., and Candler, G. V., "Predicting failure of the continuum fluid equations in transitional hypersonic flows," Phys. Fluids, Vol. 7, No. 1, Jan. 1995, pp. 210-219.

${ }^{27}$ Wang, W.-L. and Boyd, I. D., "Predicting continuum breakdown in hypersonic viscous flows," Phys. Fluids, Vol. 15, No. 1, Jan. 2003, pp. 91-100.

${ }^{28}$ Schwartzentruber, T. E., Scalabrin, L. C., and Boyd, I. D., "Investigation of Continuum Breakdown in Hypersonic Flows using a Hybrid Particle-Continuum Algorithm," AIAA-2008-4108.

${ }^{29}$ Sun, Q. and Boyd, I. D., "Evaluation of Macroscopic Properties in the Direct Simulation Method," Journal of Thermophysics and Heat Transfer, Vol. 19, No. 3, 2005, pp. 329-335. 